\title{
Simulation of free surface flows with non-hydrostatic pressure distribution
}

\author{
KRISHNA CHANDRAN ${ }^{1}$, ARUN K SAHA ${ }^{1, *}$ and PRANAB K MOHAPATRA ${ }^{2}$ \\ ${ }^{1}$ Department of Mechanical Engineering, Indian Institute of Technology Kanpur, Kanpur 208016, India \\ ${ }^{2}$ Department of Civil Engineering, Indian Institute of Technology Gandhinagar, Palaj 382355, India \\ e-mail: kchandrn@iitk.ac.in; aksaha@iitk.ac.in; pranabm@iitgn.ac.in
}

MS received 11 April 2018; revised 16 July 2018; accepted 16 August 2018; published online 4 January 2019

\begin{abstract}
In this work, a free surface flow simulator is developed in which Navier-Stokes equations using Marker and Cell (MAC) method in the framework of finite difference methodology have been solved. The free surface is tracked by the volume of fluid (VOF) method. The numerical code without free-surface is validated against the flow past a square cylinder. Three different free surface flows, i.e., dam break flow, two-dimensional cavity filling and undular bore, are studied to demonstrate the efficacy of the developed numerical model to simulate free surface flow. The numerical model used in the present work involves tracking of free surface of a single fluid in a two-fluid system. The parameters which can affect the interface orientation of the fluid is given as boundary condition at the interface. The inherent advantage of such numerical models is its ability to track free surface for high density and viscosity ratio fluids like air-water. The numerical model used in the present work is capable of solving such high density ratio two-fluid systems for which the effects due to surface tension are negligible. Results from all the problems are compared with earlier results available in literature.
\end{abstract}

Keywords. Navier-Stokes equations; volume of fluid method; free surface flow; dam break flow; undular bore.

\section{Introduction}

Free surface flows (FSF) are defined as the flows occurring in a liquid where one or more of the boundaries is not physically constrained but can adjust itself to conform to the flow condition. Mathematically, FSF require a solution in which the shape and size of the region is part of the solution [1]. Study of FSF is important in many branches of engineering. Though several analytical $[2,3]$ and experimental [4-6] models to study FSF are presented in literature, numerical modeling is extensively used to study FSF. Saint Venant Equations (SVE) wherein a uniform velocity field along the vertical plane is assumed is generally used as the governing equations to describe FSF [7]. This assumption results in a hydrostatic pressure distribution along the vertical plane. However, there are several examples of FSF where the pressure distribution is nonhydrostatic (NHP), e.g., hydraulic jump, dam break flow, flow past a sharp-crested weir, etc. In general, a FSF with stream line curvature is always with NHP. Thus, SVE should not be used to study FSF with NHP [8-10].

*For correspondence
There have been several attempts to simulate FSF with NHP. Rao [11] used Boussinesq equations (BE) to study FSF with shocks. Castro-Orgaz et al [12] with extended form of the momentum and energy equations and Hager [13] with Fawer's model studied FSF having curved streamlines. Devkota and Imberger [14] presented a Lagrangian Boussinesq model for nonlinear and dispersive FSF. Zobeyer and Steffler [15] combined the SVE and the Reynolds Averaged Navier Stokes (RANS) equations to study FSF with NHP. A separate group of models where Navier-Stokes equations are solved to study FSF with NHP is also seen in literature (table 1). The continuity and the momentum equations are solved to compute the flow field. However, the tracking of the free surface is performed separately by using an extra equation. A brief overview of several interface tracking methods are given below.

The MAC method [16] follows a staggered grid approach where the velocities are defined at the cell faces and the pressure at the cell center. Marker particles are advected in time which in turn would give the fluid configuration. The SMAC method [17] gives an improvement over the MAC method by eliminating the boundary condition difficulties. GENSMAC [18] uses the same approach as SMAC but with modified no-slip boundary condition for free surface flows in general domains. The volume of fluid method [19] emphasizes on the conservative nature of the 
Table 1. Free surface tracking methods

\begin{tabular}{|c|c|}
\hline References & Method \\
\hline Harlow and Welch (1965) [16] & $\begin{array}{l}\text { MAC (Marker and cell) } \\
\text { method }\end{array}$ \\
\hline Hirt and Shannon (1968) [34] & $\begin{array}{l}\text { MAC method with free } \\
\text { surface stress condition }\end{array}$ \\
\hline Amsden and Harlow (1970) [17] & $\begin{array}{l}\text { SMAC (Simplified marker } \\
\text { and cell) method }\end{array}$ \\
\hline Noh and Woodward (1976) [20] & $\begin{array}{l}\text { SLIC (Simplified line } \\
\text { interface calculation) }\end{array}$ \\
\hline Hirt and Nichols (1981) [19] & $\begin{array}{l}\text { VOF (Volume of fluid) } \\
\text { method }\end{array}$ \\
\hline Youngs (1982) [21] & Young's VOF \\
\hline Osher and Sethian (1988) [23] & Level set method \\
\hline Tome and McKee (1994) [18] & GENSMAC \\
\hline Sussman et al (1994) [24] & $\begin{array}{l}\text { Level set method with } \\
\text { multiphase flows }\end{array}$ \\
\hline Rudman (1997) [22] & $\begin{array}{l}\text { FCT (Flux corrected } \\
\text { transport) with VOF }\end{array}$ \\
\hline Sussman and Puckett (2000) [25] & $\begin{array}{l}\text { CLS (Coupled level set) } \\
\text { with VOF }\end{array}$ \\
\hline $\begin{array}{l}\text { (Olsson and Kreiss 2005; Van der } \\
\text { Pijl et al 2005) }[26,27]\end{array}$ & $\begin{array}{c}\text { Mass conserving level set } \\
\text { method }\end{array}$ \\
\hline
\end{tabular}

governing equations which is imperative for fluid flow simulation. A fluid is represented in terms of its volume fraction function, $F(x, y, t)$. The interface tracking becomes challenging because of the sharp jump or a discontinuity across the interface. Several interface reconstruction techniques have been developed to reduce the smearing effect caused at the interface when solved numerically, and one of the first techniques was SLIC (simple line interface calculation) by Noh and Woodward [20]. Further improvement was done by Youngs [21] when he incorporated PLIC (piecewise linear interface calculation) with VOF. Rudman [22] presented several test cases for checking the accuracy of interface tracking methods. The level set method developed by Osher and Sethian [23] and later applied on multiphase fluid flow [24], uses a continuous function to represent the interface unlike VOF which makes it easier for interface tracking. However, it has severe mass conservation problem associated with it. The advantages of both level set and VOF have been combined in [25]. Recently conservative level set methods have been proposed by Olsson and Kreiss [26], Van der Pijl et al [27]. List of all interface tracking methods discussed above is presented in table 1.

In the present study, the momentum and continuity equations are solved for only one fluid by providing dynamic interface velocity and pressure boundary conditions. The Volume of Fluid (VOF) method by Hirt and Nichols [19] is followed for surface tracking. Three different cases of FSF with NHP are studied to demonstrate the capabilities of the proposed numerical method.

\section{Governing equations}

The continuity, momentum (Navier-Stokes equations) and the volume of fluid equations constitute the governing equations for the unsteady two-dimensional, incompressible FSF.

$$
\text { Continuity equation: } \frac{\partial u^{*}}{\partial x^{*}}+\frac{\partial u^{*}}{\partial y^{*}}=0
$$

Momentum equations:

$$
\begin{aligned}
& \frac{\partial u^{*}}{\partial t^{*}}+u^{*} \frac{\partial u^{*}}{\partial x^{*}}+v^{*} \frac{\partial u^{*}}{\partial y^{*}}=-\frac{\partial p^{*}}{\partial x^{*}}+\frac{1}{R e}\left(\frac{\partial^{2} u^{*}}{\partial x^{* 2}}+\frac{\partial^{2} u^{*}}{\partial y^{* 2}}\right) \\
& \frac{\partial v^{*}}{\partial t^{*}}+u^{*} \frac{\partial v^{*}}{\partial x^{*}}+v^{*} \frac{\partial v^{*}}{\partial y^{*}}=-\frac{\partial p^{*}}{\partial y^{*}}+\frac{1}{R e}\left(\frac{\partial^{2} v^{*}}{\partial x^{* 2}}+\frac{\partial^{2} v^{*}}{\partial y^{* 2}}\right)+\frac{g^{*}}{F r^{2}}
\end{aligned}
$$

$$
\text { Volume of fluid equation: } \frac{d F}{d t^{*}}+u^{*} \frac{\partial F}{\partial x^{*}}+v^{*} \frac{\partial F}{\partial y^{*}}=0
$$

Equations (1) - (3) are in non-dimensional form, where, the non-dimensional parameters are: $x^{*}=\frac{x}{L}, y^{*}=\frac{y}{L}, u^{*}=$ $\frac{u}{U}, v^{*}=\frac{v}{U}, p^{*}=\frac{p}{\rho U^{2}}, g^{*}=\frac{g_{y}}{g}=-1.0, t^{*}=\frac{t}{T}$ and $T=\frac{L}{U}$ with $x$ and $y$ as the horizontal and vertical directions, respectively; $L$ and $U$ are the length and the velocity scales, respectively; $g$ is the acceleration due to gravity; $R e=\frac{U L}{v}$ is the Reynolds number and $F r=\frac{U}{\sqrt{g L}}$ is the Froude number.

Equation (3) represents volume conservation. The variable $F$ is a time dependent scalar function and is defined as the fraction of a cell occupied by the fluid. Thus, the fluid region is indicated by $F=1$ and a void region has $F=0$. A free surface cell is indicated when $0<F<1$. The governing equations are solved numerically in the non-dimensional form and the superscript $(*)$, used to represent the non-dimensional terms is dropped in rest of the presentations for simplicity.

\section{Numerical solution}

The computational domain is divided into a set of rectangular cells and a staggered grid arrangement is used. The numerical solution of Navier-Stokes equation uses the following strategy (figure 1).

- In the predictor step Eq. (2) is discretized as [28]

$$
\begin{aligned}
& \frac{u_{i, j}^{p}-u_{i, j}^{n}}{\Delta t}=-\left(\frac{p_{i+1, j}^{n}-p_{i, j}^{n}}{\Delta x_{i}}\right)+\frac{3}{2} G_{x}(u, v)^{n}-\frac{1}{2} G_{x}(u, v)^{n-1} \\
& \frac{v_{i, j}^{p}-v_{i, j}^{n}}{\Delta t}=-\left(\frac{p_{i, j+1}^{n}-p_{i, j}^{n}}{\Delta y_{j}}\right)+\frac{3}{2} G_{y}(u, v)^{n}-\frac{1}{2} G_{y}(u, v)^{n-1} \\
& G_{x}=-\left(u \frac{\partial u}{\partial x}+v \frac{\partial u}{\partial y}\right)+\frac{1}{\operatorname{Re}}\left(\frac{\partial^{2} u}{\partial x^{2}}+\frac{\partial^{2} u}{\partial y^{2}}\right) \\
& G_{y}=-\left(u \frac{\partial v}{\partial x}+v \frac{\partial v}{\partial y}\right)+\frac{1}{\operatorname{Re}}\left(\frac{\partial^{2} v}{\partial x^{2}}+\frac{\partial^{2} v}{\partial y^{2}}\right)-\frac{1.0}{F r^{2}}
\end{aligned}
$$




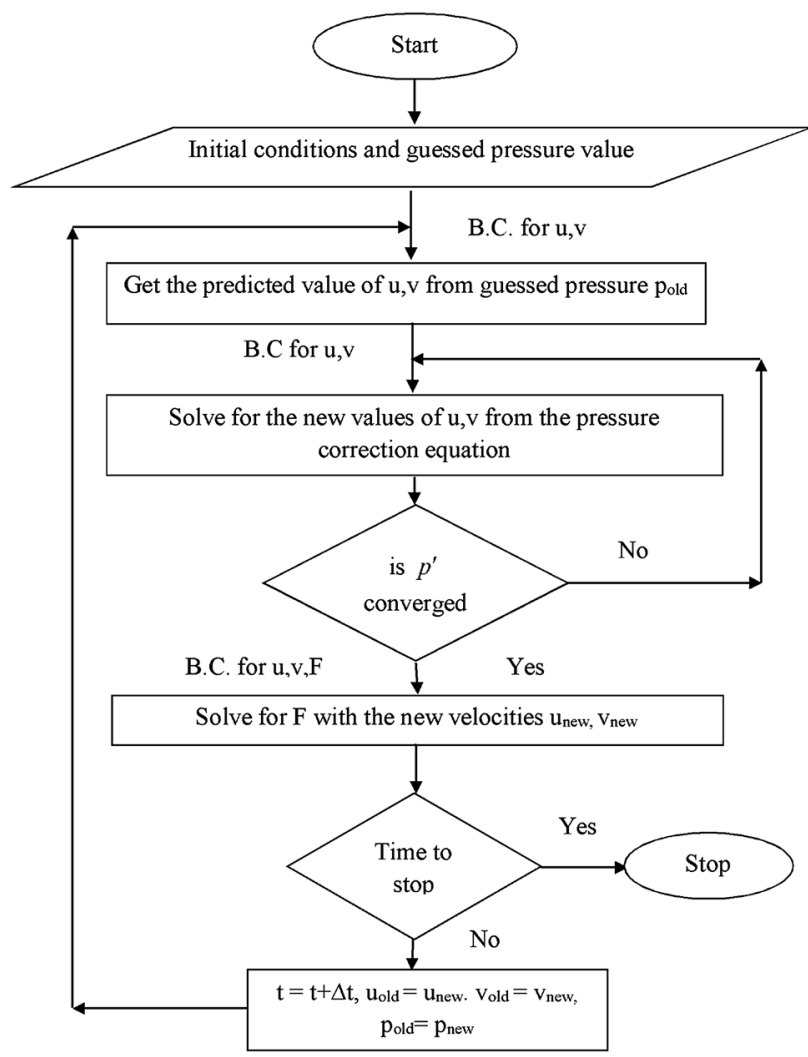

Figure 1. Flow chart of the numerical algorithm.

where $G_{x}$ and $G_{y}$ contains both the advection and diffusion terms. The superscripts $(p, n, n-1)$ represent the predicted, present time level and previous time level values, respectively. The complete discretization of $G_{x}$ and $G_{y}$ terms are given in [29]. Equation (4) represents the second-order Adams-Bashforth time marching where the first time level values are found using Euler explicit method.

- Continuity equation results in Poisson equation for pressure correction, $p^{\prime}$

$$
\frac{\partial^{2} p^{\prime}}{\partial x^{2}}+\frac{\partial^{2} p^{\prime}}{\partial y^{2}}=\frac{1}{\Delta t}\left(\frac{\partial u}{\partial x}+\frac{\partial v}{\partial y}\right)
$$

Discretization of Eq. (5) results in

$$
\begin{gathered}
\frac{p_{i-1, j}^{\prime}-2 p_{i, j}^{\prime}+p_{i+1, j}^{\prime}}{\Delta x_{i}^{2}}+\frac{p_{i, j-1}^{\prime}-2 p_{i, j}^{\prime}+p_{i, j+1}^{\prime}}{\Delta y_{j}^{2}} \\
=\frac{1}{\Delta t}\left[\frac{u_{i, j}^{p}-u_{i-1, j}^{p}}{\Delta x_{i}}+\frac{v_{i, j}^{p}-v_{i, j-1}^{p}}{\Delta y_{j}}\right]
\end{gathered}
$$

which can be written as [30] (by dropping neighbouring pressure correction terms)

$$
\begin{gathered}
p_{i, j}^{\prime}=-r_{0}\left(\frac{u_{i, j}^{p}-u_{i-1, j}^{p}}{\Delta x_{i}}+\frac{v_{i, j}^{p}-v_{i, j-1}^{p}}{\Delta y_{j}}\right) / \\
{\left[2 \Delta t\left\{\frac{1}{\left(\Delta x_{i}\right)^{2}}+\frac{1}{\left(\Delta y_{j}\right)^{2}}\right\}\right]}
\end{gathered}
$$

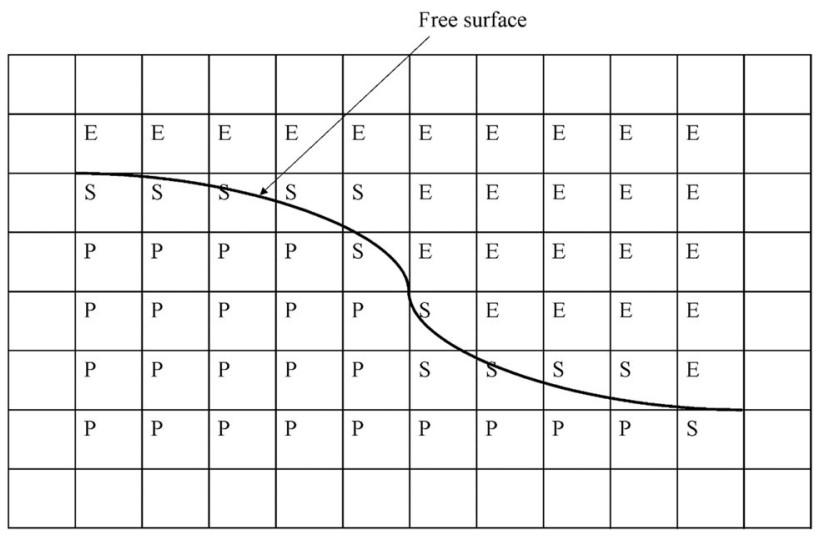

Figure 2. Cell types used for the present problem. $\mathrm{P}$ denotes a full cell $(F=1), \mathrm{S}$ denotes a surface cell $(0<F \leq 1)$ and $\mathrm{E}$ denotes an empty cell $(F=0)$.

In the present work, Eq. (7) is solved using point GaussSeidel technique with over relaxation parameter $r_{o}=1.8$ to accelerate the iteration process.

- The final solution for the velocity and pressure are given as [16]:

$$
\begin{gathered}
p_{i, j}^{n+1} \leftarrow p_{i, j}^{n}+p_{i, j}^{\prime} \\
u_{i, j}^{n+1} \leftarrow u_{i, j}^{p}+\frac{\Delta t}{\Delta x_{i}} p_{i, j}^{\prime} \text { and } v_{i, j}^{n+1} \leftarrow v_{i, j}^{p}+\frac{\Delta t}{\Delta y_{j}} p_{i, j}^{\prime}
\end{gathered}
$$

- The corrected velocity field is used to solve Eq. (3) for evolution of the free surface.

\subsection{Free surface tracking}

Equation (3) is solved by donor-acceptor method of Hirt and Nichols [19], where volume fractions are converted to volume fluxes. Figure 2 depicts the notation used to identify a full cell (P) a surface cell (S) and an empty cell E. The interface is a surface cell with neighbouring cells being full cells, surface cells or empty cells. Full cell $(\mathrm{P})$ has $F=1$ while empty cell has $F=0$. The surface cells (S) are identified with volume fraction in the range $0<F \leq 1$. The velocity boundary conditions at the interface are interpolated based on different types of surface cells as categorized in table 2. Once the velocities at the surface cells are estimated, the volume fraction equation is solved. Figure 3 shows different types of interface orientation. The dashed line in figure 3 (a) restricts the amount of volume flux to be fluxed across the cell face from the donor cell to the acceptor cell. The volume flux across the cell face is calculated in the same manner as given in [19]. Figures 3(b) and (c) show the interface orientation in horizontal and vertical directions, respectively. Figure 3(d) shows a horizontal interface orientation for the donor cell while the acceptor cell is completely empty. In such situations, the whole of the fluid 
Table 2. Types of surface cells [31].

\begin{tabular}{|c|c|c|c|c|c|}
\hline \multirow[b]{2}{*}{ Cell type } & \multicolumn{4}{|c|}{ Cell indices } & \multirow[b]{2}{*}{ Velocity interpolation } \\
\hline & $i, j+1$ & $\mathrm{i}+1, \mathrm{j}$ & $\mathrm{i}, \mathrm{j}-1$ & $\mathrm{i}-1, \mathrm{j}$ & \\
\hline Type-1 & $0<F<1$ & $F=0$ & $0<F<1$ & $F=1$ & $u_{i, j}=u_{i-1, j}-\frac{\Delta x_{i}}{\Delta y_{j}}\left(v_{i, j}-v_{i, j-1}\right)$ \\
\hline Type-2 & $0<F<1$ & $F=0$ & $0<F<1 / F=1$ & $F=0$ & $u_{i, j}=u_{i-1, j}=0$ \\
\hline Type-3 & $F=0$ & $F=0$ & $0<F<1 / F=1$ & $0<F<1 / F=1$ & $u_{i, j}=u_{i-1, j}, v_{i, j}=v_{i, j-1}$ \\
\hline Type-4 & $F=0$ & $F=0$ & $F=0$ & $0<F<1 / F=1$ & $\begin{array}{l}v_{i, j}=v_{i-1, j} \\
v_{i, j-1}=v_{i-1, j-1} \\
u_{i, j}=u_{i-1, j}-\frac{\Delta x_{i}}{\Delta y_{j}}\left(v_{i, j}-v_{i, j-1}\right)\end{array}$ \\
\hline \multirow[t]{2}{*}{ Special type } & \multicolumn{4}{|c|}{ Cell indices } & Velocity interpolation \\
\hline & $0<F<1 / F=1$ & $\begin{array}{l}\mathrm{i}, \mathrm{j}+1 \\
F=0\end{array}$ & $\begin{array}{c}\mathrm{i}+1, \mathrm{j} \\
0<F<1 / F=1\end{array}$ & $\begin{array}{c}\mathrm{i}+1, \mathrm{j}+1 \\
F=0\end{array}$ & $u_{i, j+1}=u_{i, j}$ \\
\hline
\end{tabular}

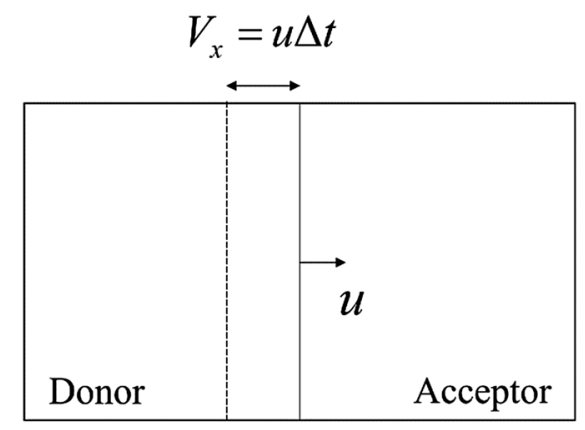

(a)

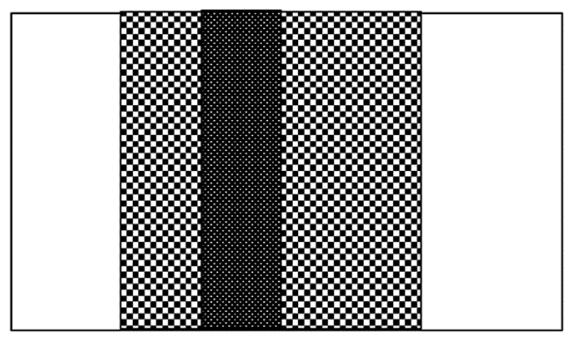

(c)

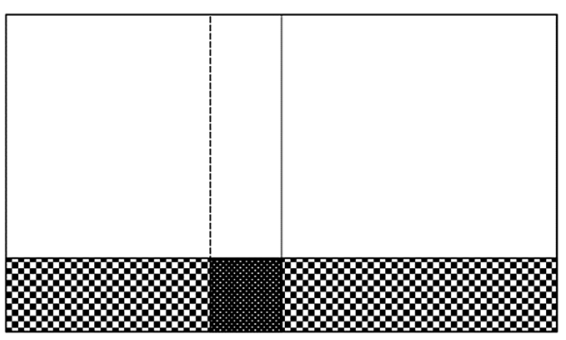

(b)

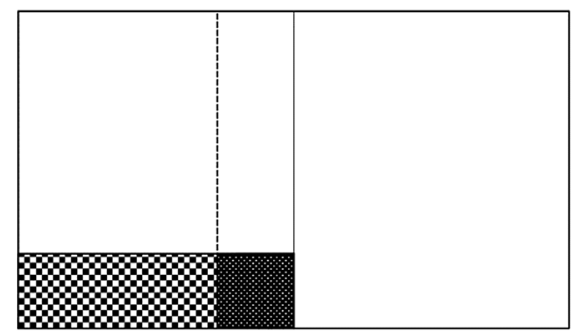

(d)

Figure 3. Examples of interface orientation in a surface cell. The total volume advected is shown by the dashed line (a). Interfaces are either horizontal (b) and (d) or vertical (c). The dark portion within the dashed line gives the volume fraction of fluid advected.

fraction (darkened area) within the dashed line is advected to the acceptor cell. The fluxes across the cell faces depend on the sign of the velocity normal to the cell face. The new values of $F$ can be found out by applying the conservation law as presented below.

The net rate of change of flux $=$ flux in - flux out

$$
\begin{aligned}
\operatorname{vol}\left(F_{i, j}^{n+1}\right)= & \operatorname{vol}\left(F_{i, j}^{n}\right)+\operatorname{sign}\left(u_{i-1, j}^{n+1}\right)\left(\delta F_{x l}\right) \\
& +\operatorname{sign}\left(v_{i, j-1}^{n+1}\right)\left(\delta F_{y b}\right)-\operatorname{sign}\left(u_{i, j}^{n+1}\right)\left(\delta F_{x r}\right) \\
& -\operatorname{sign}\left(v_{i, j}^{n+1}\right)\left(\delta F_{y t}\right)
\end{aligned}
$$

where $\delta F_{x l}, \delta F_{x r}, \delta F_{y b}, \delta F_{y t}$ are the amount of fluxes across the left, right, bottom and top faces, respectively.

The new $F$ value is then given by

$$
F_{i, j}^{n+1}=\frac{\operatorname{vol}\left(F_{i, j}^{n+1}\right)}{\Delta x_{i} \Delta y_{j}}
$$

The interface representation within a surface cell and the interpolation of cell center pressure from the free surface pressure is performed [19]. In the present method two types of orientation of the surface within a cell are considered, the surface is either vertical or horizontal. It is important to 
know the location of the boundary within a surface cell for the accurate computation of boundary conditions. The boundary is approximated by a straight line cutting through the cell and then it is moved across the cell to a position where it intersects with the amount of $\mathrm{F}$ value contained in that cell [19]. To know whether the boundary orientation is vertical or horizontal within a cell, the boundary slope should be known and for that the boundary is represented by two single valued functions $H(x)$ and $B(y)$.

$$
\begin{aligned}
H_{i} & =F_{i, j-1} \Delta y_{j-1}+F_{i, j} \Delta y_{j}+F_{i, j+1} \Delta y_{j+1} \\
B_{j} & =F_{i-1, j} \Delta x_{i-1}+F_{i, j} \Delta x_{i}+F_{i+1, j} \Delta x_{i+1}
\end{aligned}
$$

$\left.\frac{d H}{d x}\right|_{i}$

$=\frac{\left(H_{i+1} \Delta x_{i}+H_{i} \Delta x_{i+1}\right) \times d x x l-\left(H_{i-1} \Delta x_{i}+H_{i} \Delta x_{i-1}\right) \times d x x r}{2 \Delta x_{i} \times d x x l \times d x x r}$

$$
\begin{aligned}
& \left.\frac{d B}{d y}\right|_{j} \\
& =\frac{\left(B_{j+1} \Delta y_{j}+H_{j} \Delta y_{j+1}\right) \times d y y b-\left(H_{j-1} \Delta y_{j}+H_{j} \Delta y_{j-1}\right) \times d y y t}{2 \Delta y_{i} \times d y y b \times d y y t}
\end{aligned}
$$

$d x x l=0.5 \times\left(\Delta x_{i}+\Delta x_{i-1}\right), \quad d x x r=0.5 \times\left(\Delta x_{i}+\Delta x_{i+1}\right)$ $d y y b=0.5 \times\left(\Delta y_{j}+\Delta y_{j-1}\right), d y y t=0.5 \times\left(\Delta y_{j}+\Delta y_{j+1}\right)$

Equations (12) and (13) give a good approximation for the slope of the boundary within an interface cell. If $\left|\frac{d H}{d x}\right|<\left|\frac{d B}{d y}\right|$ then the boundary is more horizontal than vertical and if $\left|\frac{d B}{d y}\right|<\left|\frac{d H}{d x}\right|$ then the boundary is more vertical than horizontal. The smaller derivative gives the best approximation since this implies that the variation of $\mathrm{H}$ or $\mathrm{B}$ along $\mathrm{x}$ or $\mathrm{y}$ direction is gradual and hence the normal to the interface lies in a direction where $\mathrm{F}$ value variation is steeper.

For fluid flow problems involving free surface, the pressure at the surface of the fluid is given as zero. The surface boundary within an interface cell can be located at a fraction of cell size ( $\Delta \mathrm{x}$ or $\Delta \mathrm{y})$ away from the cell centre. The cell centre pressure is interpolated from the imposed zero surface cell pressure and with the neighbouring cell which contains more fluid. Equations (12) and (13) are used to find which neighbouring cell contains more fluid. Figure 4 shows how the interface cell centre pressure is interpolated with the neighbouring fluid cell.

For an interface cell, if $\left|\frac{d H}{d x}\right|<\left|\frac{d B}{d y}\right|$ then the interface is mostly horizontal than vertical and if for that cell $\frac{d B}{d y}<0$ then, Eq. (13) shows that $B_{j-1}>B_{j+1}$. This shows that $\mathrm{F}$ value in the $(j-1)$ th cell is more than that of the $(j+1)$ th cell. Hence more fluid lies below the interface cell $(\mathrm{i}, \mathrm{j})$. The

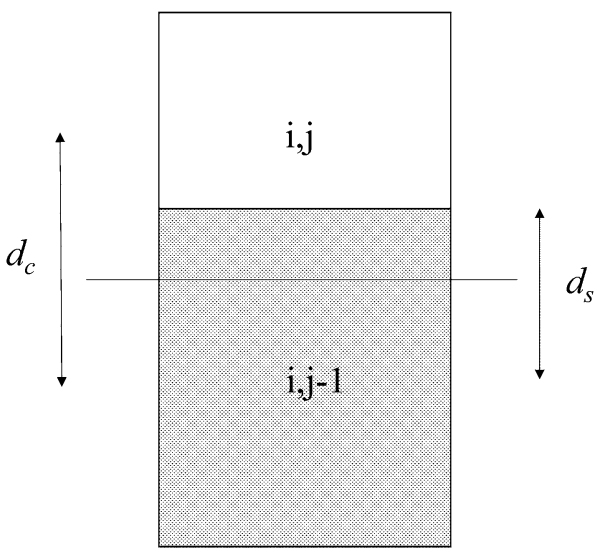

Figure 4. Surface pressure interpolation from a full cell $(i, j-1)$ to a surface cell $(\mathrm{i}, \mathrm{j})$.

surface pressure $p_{s}$, as mentioned above is taken as zero. The cell centre pressure $p_{i, j}$ is found out by finding the interpolation factor $\eta$ and then interpolating with the full fluid cell pressure $p_{i, j-1}$ which lies below as shown in figure 4. The interpolation factor $\eta$ is given by the ratio of cell centre distance of the surface or interface cell and the full cell to the distance between the cell centre of the full cell to the surface of the fluid.

$$
\eta=\frac{d_{s}}{d_{c}}
$$

In the case as shown in figure $4 d_{c}=0.5 \times\left(\Delta y_{j}+\right.$ $\left.\Delta y_{j-1}\right), d_{s}=F_{i, j} \times \Delta y_{j}+0.5 \times \Delta y_{j-1}$. The pressure at cell centre is interpolated by using a linear interpolation as give below

$$
p_{i, j}=p_{i, j-1}+\eta \times\left(p_{s}-p_{i, j-1}\right)
$$

\subsection{Stability}

In order to prevent numerical instabilities in the simulation, the time increment is determined subjected to two restrictions, namely, the Courant-Friedrich-Lewy (CFL) condition and restriction due to grid Fourier numbers. According to CFL condition, the distance travelled by the fluid particles in one time increment should not be more than one space increment. This leads to the following constraint condition

$$
\Delta t<\min \left\{\frac{\Delta x_{i}}{\left|u_{i, j}^{n+1}\right|}, \frac{\Delta y_{j}}{\left|v_{i, j}^{n+1}\right|}\right\}
$$

The restriction imposed by the grid Fourier number becomes dominant when the viscous diffusion terms are more important. This condition is given by

$$
\Delta t<\min \left[\frac{\operatorname{Re}}{2}\left\{\frac{\left(\Delta x_{i}\right)^{2}\left(\Delta y_{j}\right)^{2}}{\left(\left(\Delta x_{i}\right)^{2}+\left(\Delta y_{j}\right)^{2}\right)}\right\}\right]
$$


Table 3. Input parameters.

\begin{tabular}{|c|c|c|c|}
\hline $\begin{array}{l}\text { Model } \\
\text { problems }\end{array}$ & Dimensions & Grid/Mesh size & $\begin{array}{c}\text { Non- } \\
\text { dimensional } \\
\text { numbers }\end{array}$ \\
\hline $\begin{array}{l}\text { Dam } \\
\text { break } \\
\text { flow }\end{array}$ & $\begin{array}{c}\text { base width of } \\
\text { dam }=1.0 \\
\text { height of } \\
\text { dam }=2.0\end{array}$ & $\begin{array}{c}\Delta \mathrm{x}=\Delta \mathrm{y}=0.1 \\
\Delta \mathrm{x}=\Delta \mathrm{y}=0.05 \\
\Delta \mathrm{x}=\Delta \mathrm{y}=0.025 \\
\Delta \mathrm{t}=0.001\end{array}$ & $\begin{array}{c}R e=30000 \\
F r=1.0\end{array}$ \\
\hline $\begin{array}{l}\text { 2-D } \\
\text { cavity } \\
\text { filling }\end{array}$ & $\begin{array}{l}3.375 \times 2.5 \text { in } \\
\text { width by } \\
\text { height. } \\
\text { Opening }=1.0\end{array}$ & $\begin{array}{c}54 \times 40 \text { cells width } \\
\text { by height. } \\
\text { Opening }=16 \\
\text { cells wide. } \\
\Delta \mathrm{t}=0.001\end{array}$ & $\begin{array}{c}R e=15 \\
F r=0.577\end{array}$ \\
\hline $\begin{array}{l}\text { Undular } \\
\text { bore }\end{array}$ & $\begin{array}{c}10 \times 2 \text { in length } \\
\text { by height }\end{array}$ & $\begin{array}{c}100 \times 40 \text { uniform } \\
\text { mesh cells } \\
\Delta \mathrm{t}=0.001\end{array}$ & $\begin{array}{l}\operatorname{Re}=4.33 \\
F r=0.31\end{array}$ \\
\hline
\end{tabular}

The final value of time increment is taken as the minimum of the above two restrictions. Since the CFL restriction is derived approximating the advective terms in first order, and the present discretization scheme is second order, a weightage factor (less than 1.0) depending on the value of Reynolds number has been used in the present study. Typically, the time step used in the computation is of the order of $10^{-3}$.

Laminar flow past a square cylinder has been simulated and compared with the results of Sohankar et al [32] to validate the Navier-Stokes solver. The detailed validation can be found in Saha et al [28].

\section{Results and discussion}

The proposed numerical model is validated for laminar flow past square cylinder. In addition, three different free surface flows with non-hydrostatic pressure distributions viz., dam break flow, 2-D cavity filling and undular bore are studied to demonstrate the capabilities of the numerical model. Various input parameters and boundary conditions for all problems considered are presented in tables 3 and 4, respectively.

\subsection{Dam break flow}

A rectangular column of static water is confined between two plates. Therefore, the pressure distribution is hydrostatic, initially. At time $\mathrm{t}=0$, the right plate (considered as a dam) is removed instantaneously and the propagation of the fluid is simulated.

Figure 5 shows the surface profiles of the dam break flow at various non-dimensional times. The flood wave propagates because of the head difference between two sides of the dam. Pressure distribution becomes non-hydrostatic just as the dam breaks.

Propagation of the surge front of the dam break flow are found to be matching almost satisfactorily with that of the experimental results of Martin and Moyce [3] and Hirt and Nichols [19] (figure 6). Initially, the surge velocity is zero and then it increases and attains a constant value of around 2.0 which is also the maximum velocity attainable by the conservation of energy.

Table 4. Boundary conditions.

\begin{tabular}{|c|c|c|c|c|c|}
\hline & Left & Right & Top & Bottom & Obstacle walls \\
\hline Square cylinder & $\begin{array}{l}u=1 \\
v=0\end{array}$ & $\begin{array}{l}u=\text { Orlanski }[28] \\
\frac{\partial v}{\partial x}=0\end{array}$ & $\begin{array}{l}\frac{\partial u}{\partial y}=0 \\
v=0\end{array}$ & $\begin{array}{l}\frac{\partial u}{\partial y}=0 \\
v=0\end{array}$ & $\begin{array}{l}u=0 \\
v=0\end{array}$ \\
\hline Dam break & $\begin{aligned} u=0, \frac{\partial v}{\partial x} & =0 \\
\frac{\partial F}{\partial x} & =0\end{aligned}$ & $\begin{aligned} \frac{\partial u}{\partial x}=0, \frac{\partial v}{\partial x} & =0 \\
\frac{\partial F}{\partial x} & =0\end{aligned}$ & $\begin{aligned} \frac{\partial u}{\partial y}=0, v & =0 \\
\frac{\partial F}{\partial y} & =0\end{aligned}$ & $\begin{array}{r}\frac{\partial u}{\partial y}=0, v=0 \\
\frac{\partial F}{\partial y}=0\end{array}$ & - \\
\hline Cavity filling & $\begin{array}{l}u=0, \frac{\partial v}{\partial x}=0 \\
\frac{\partial F}{\partial x}=0\end{array}$ & $\begin{array}{l}u=0, \frac{\partial v}{\partial x}=0 \\
\frac{\partial F}{\partial x}=0\end{array}$ & $\begin{array}{l}\frac{\partial u}{\partial y}=0, v=0 \\
\frac{\partial F}{\partial y}=0\end{array}$ & $\left.\begin{array}{rl}u=0, v & =0 \\
\frac{\partial F}{\partial y} & =0\end{array}\right\}$ at walls & - \\
\hline Undular bore & $\begin{array}{l}\left.\begin{array}{l}u=1, v=0 \\
F=1\end{array}\right\} \begin{array}{l}\text { at } \\
\text { bore-inlet }\end{array} \\
\left.\begin{array}{rl}\frac{\partial u}{\partial x}=0, \frac{\partial v}{\partial x}=0 \\
\frac{\partial F}{\partial x}=0\end{array}\right\} \begin{array}{l}\text { above } \\
\text { bore-inlet }\end{array}\end{array}$ & $\begin{array}{l}u=0, \frac{\partial v}{\partial x}=0 \\
\frac{\partial F}{\partial x}=0\end{array}$ & $\begin{array}{l}\frac{\partial u}{\partial y}=0, v=0 \\
\frac{\partial F}{\partial y}=0\end{array}$ & $\begin{array}{l}\frac{\partial u}{\partial y}=0, v=0 \\
\frac{\partial F}{\partial y}=0\end{array}$ & - \\
\hline
\end{tabular}



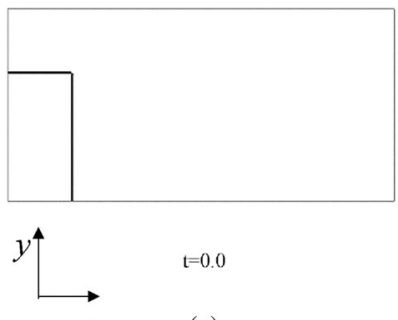

(a)

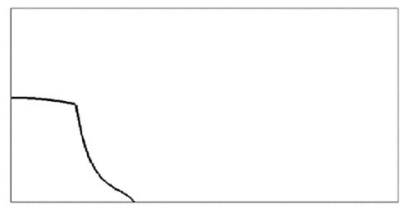

$\mathrm{t}=0.8$

(b)

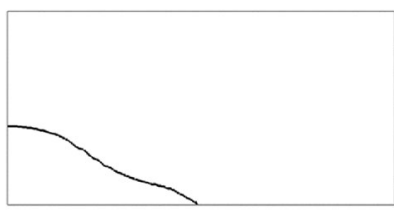

$\mathrm{t}=1.4$

(c)

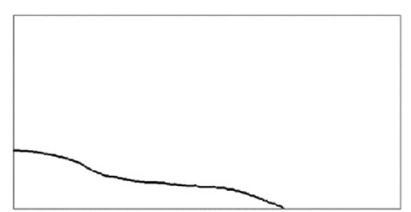

$\mathrm{t}=2.0$

(d)
Figure 5. Surface profile of broken dam at different times. The dark line shows the air-water interface at different time instants.

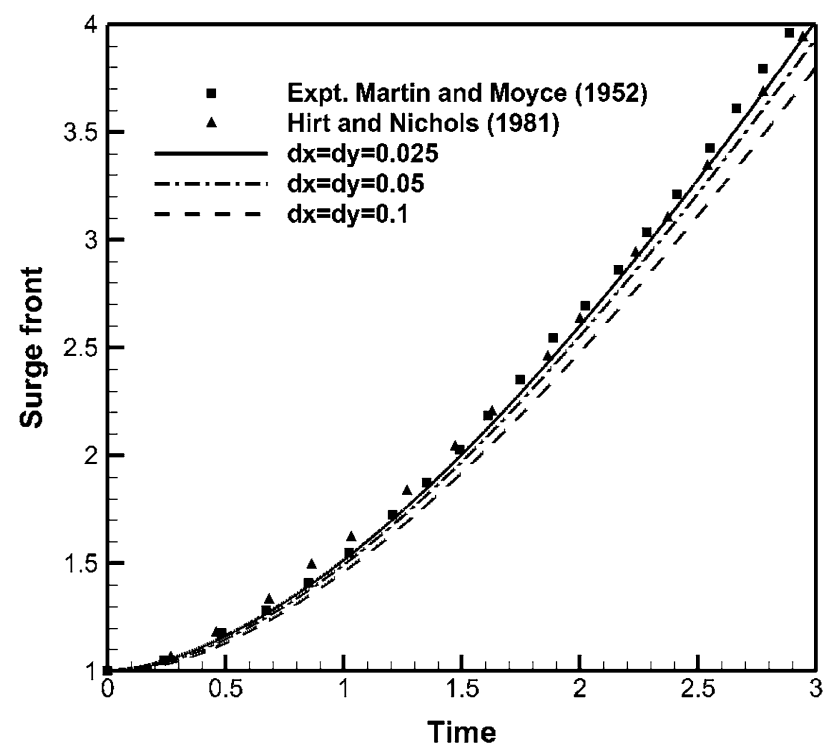

Figure 6. Surge front variation with time. Results are compared with literature [5] for both coarse and fine grids.

However, attainment of the constant velocity of the surge front may be attributed to high $R e$ which is close to an inviscid flow assumption.

Figure 7 shows the comparison of surge front variation with time for the dam break flow example problem given in OpenFoam. The result shows a good match with coarse as well as with fine mesh. CPU time is slightly better compared to OpenFoam as shown in table 5. This is due to the one-fluid approach used in the present solver compared to the multi-fluid approach used in OpenFoam.

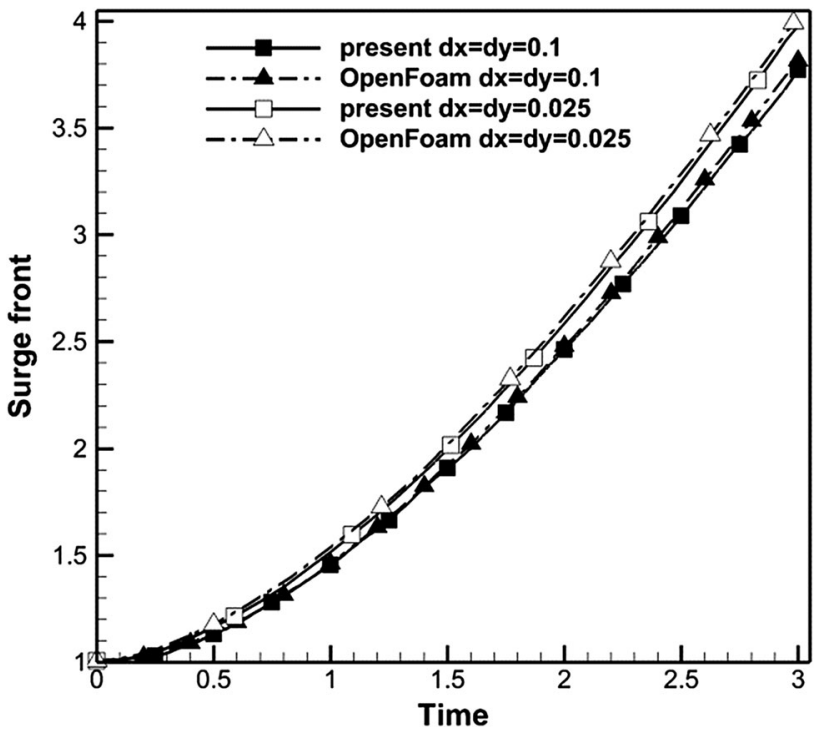

Figure 7. Comparison of surge front variation with time for the present work and the multi-fluid approach of OpenFoam.

Table 5. Comparison of CPU time with OpenFoam results for dam break flow problem.

\begin{tabular}{lcc}
\hline Grid size & $\begin{array}{c}\text { Present [CPU time } \\
(\mathrm{s})]\end{array}$ & $\begin{array}{c}\text { OpenFoam [CPU time } \\
(\mathrm{s})]\end{array}$ \\
\hline$\Delta x=\Delta y=0.1$ & 8.4 & 10.3 \\
$\Delta x=\Delta y=0.025$ & 12.2 & 13.4 \\
\hline
\end{tabular}

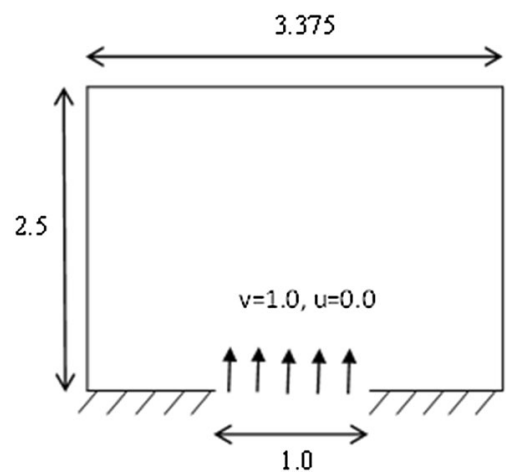

Figure 8. Layout of a 2-D rectangular cavity. Fluid enters from the bottom of the cavity.

\subsection{2-D cavity filling}

Figure 8 shows a 2-D rectangular cavity in which fluid enters from the bottom through an opening. $R e$ is defined by using the width of the opening cavity as the length scale, and velocity scale $U=1.0$. Results from the present numerical model are matched at certain non-dimensional 

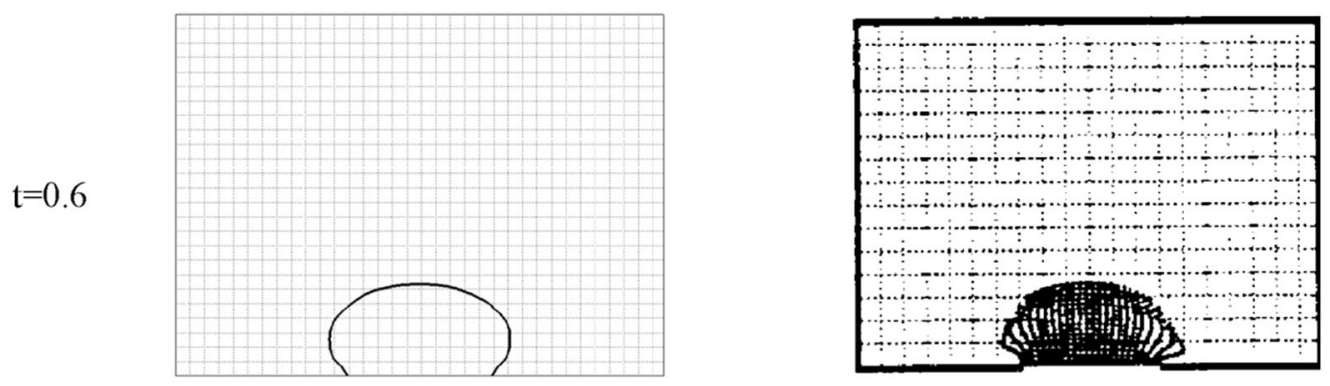

(a)
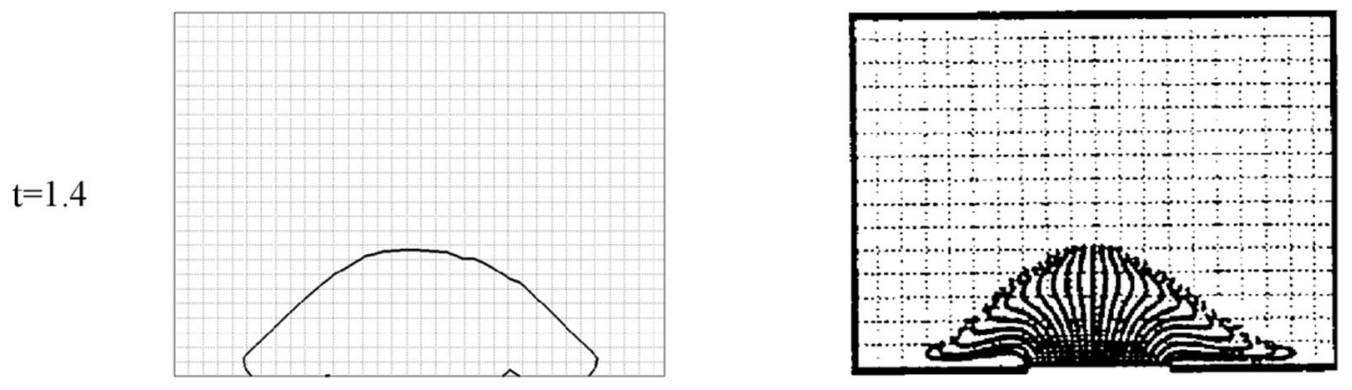

(b)
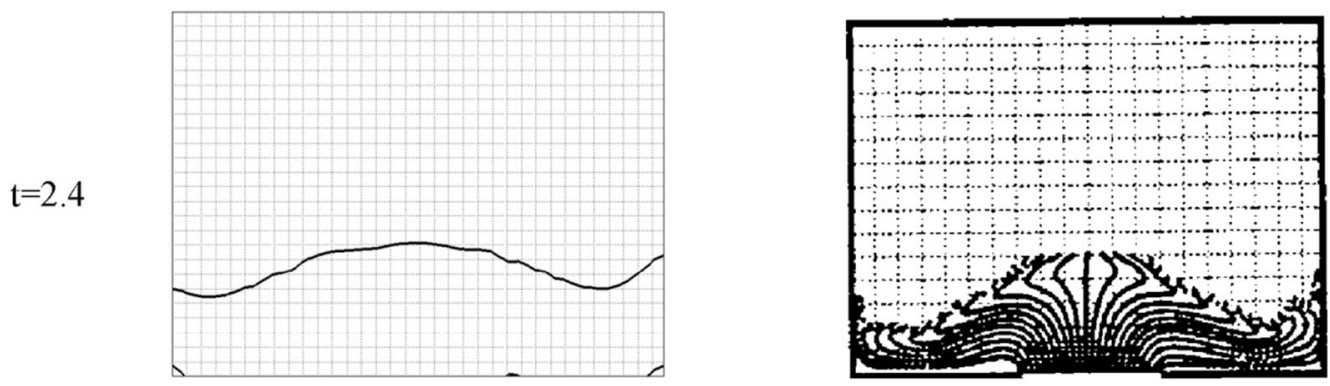

(c)

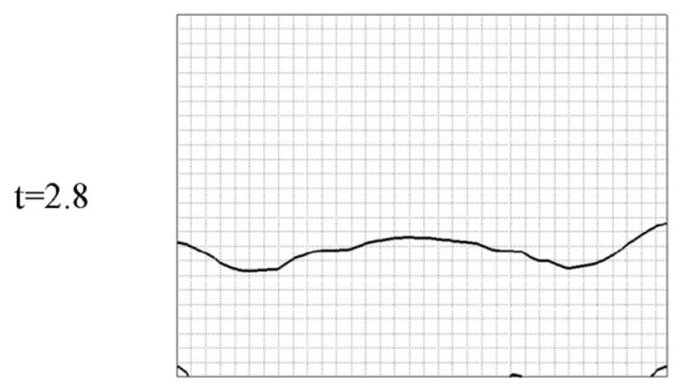

Present

(d)

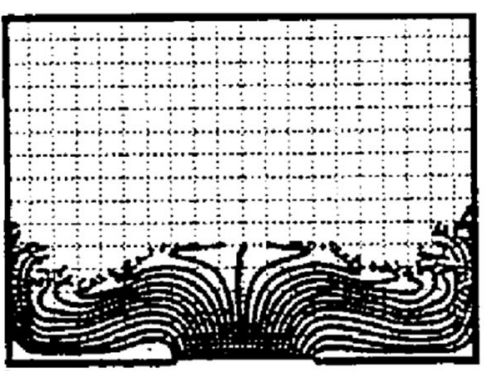

SMAC

Figure 9. Cavity filling at different times. Results are qualitatively matched with the literature [33].

times with those obtained by Chen et al [33] by using SMAC method (figure 9). One advantage of using VOF over SMAC is in computational time and memory which is more for SMAC because of the marker particles which are used for tracking the interface. The surface profile remains smooth for low $R e$ and it is distorted as $R e$ is increased. The distortion of the surface was also observed as $\mathrm{Fr}$ is decreased. These characteristics were also reported by Chen et al [33].

\subsection{Undular bore}

A step wave or a bore is created when a horizontal layer of fluid is pushed against a rigid vertical wall and it moves in a 


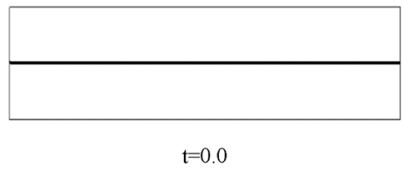

(a)

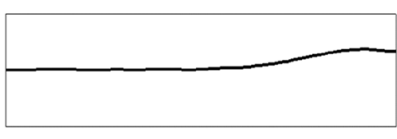

$\mathrm{t}=0.8$

(c)

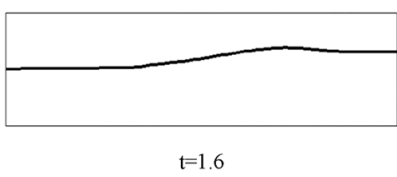

(e)

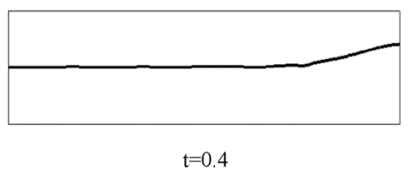

(b)

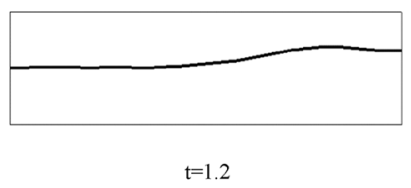

(d)

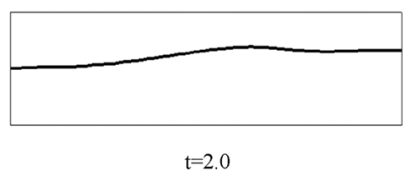

(f)
Figure 10. Undular bore propagation against a wall at various time instants for $R e=4.33$ and $F r=0.44$. The fluid enters at constant velocity.

direction away from the wall (figure 10). A bore front having a well-behaved, undular shape, is created if the velocity with which the fluid hits the vertical wall is not too high. However, the bore breaks and is highly irregular if the incident velocity is too high. A non-dimensional bore height of 1.4 is matched with Hirt and Shannon [34] for non-dimensional numbers given in table 3 . This problem has also been tested to demonstrate the diffusive nature of first order upwind scheme against the donor-acceptor method which is used in the present study.

Figure 10 shows the undular bore propagation with time from an initial bore height (non-dimensional) of unity. The shape of the bore is maintained because of small values of the Reynolds number and the Froude number. The bore loses its shape by increasing the Froude number and it results in a breaking bore. Note that though the surface pressure is given 0.0. A free surface stress condition [24] has also been tested and is shown to give the same bore height. However, the main objective of using free surface stress condition is to show that the bore satisfies the conservation of mass and momentum [4], so that the bore height and the bore velocity match with the theoretical values. Figure 11(a) shows the variation of the bore height at a location near to the vertical wall, with time for various $R e=4.33,10,30$ and 80 with $F r=0.44$. For low $R e$, a significant change in the maximum height achieved by the corresponding bores has been observed. However, the difference in maximum height becomes marginal and a wavy nature is observed downstream of the bore as $R e$ is increased. Figure 11(b) shows the variation of the bore height with time for donor acceptor (DOAC) and first order upwind (FOU) scheme. The figure clearly reveals the diffusive nature of first order upwind scheme when used in the discretized volume fraction equation. The bore height diffuses upwards in the $y$-direction for FOU scheme while DOAC scheme prevents such numerical diffusion thus retaining a sharp interface.

Note that the pressure distribution is non-hydrostatic in all the three problems discussed above.
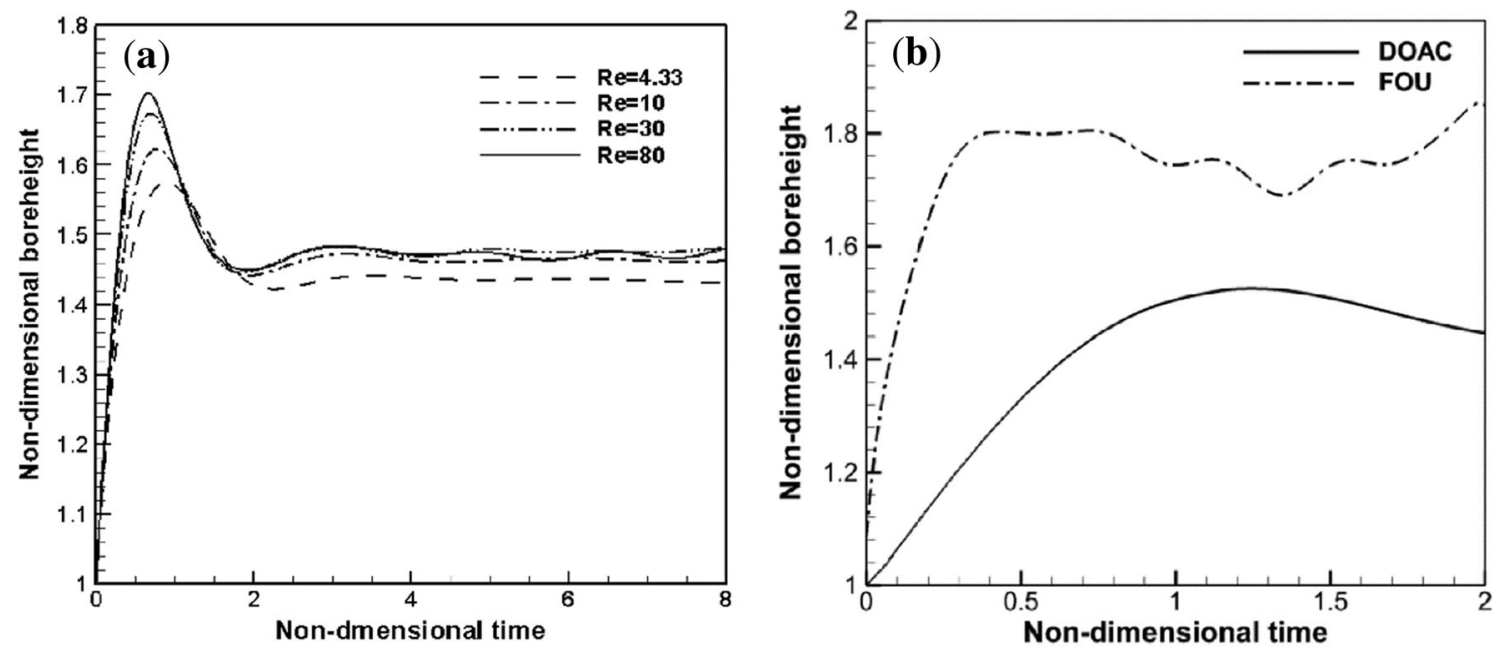

Figure 11. (a) Bore height variation with time for various Reynolds numbers with $F r=0.44$. (b) Comparison of bore height variation with time between donor acceptor (DOAC) scheme and first order upwind (FOU) advection scheme for volume fraction equation at $R e=4.33$ and $F r=0.44$. 


\section{Conclusions}

A numerical model to study free surface flows with nonhydrostatic pressure distribution in a vertical plane has been proposed in the present study. Navier-Stokes equations along with the continuity equations have been numerically solved by a finite difference approach using the Marker and Cell (MAC) method to compute the flow field. The free surface is tracked by the volume of fluid (VOF) method. Laminar flow past a square cylinder has been simulated to validate the Navier-Stokes solver. In addition, three different free surface flows with non-hydrostatic pressure distributions, i.e., dam break flow, two-dimensional cavity filling and undular bore, have been studied to demonstrate the capabilities of the developed numerical model. Results from all the problems have been compared with earlier results available in literature. Simulated results show that the proposed numerical model could be used to study free surface flows with non-hydrostatic pressure distributions. The main limitation of the solver is in number of interface orientations. In the present work, the interface is considered either purely vertical or purely horizontal. Oblique orientation of the interface within a surface cell will add more robustness. This will reduce the number of cells required to capture a sharp interface and hence reduce the computational time too.

Given the robustness of the method, the following problems can be taken up in the future; (i) addition of surface tension models in the solver for surface tension dominant flows, (ii) addition of turbulent models for high $R e$ free surface turbulent flows, etc.

\section{List of symbols}

$\begin{array}{ll}F & \text { volume fraction of fluid } \\ g & \text { acceleration due to gravity } \\ \mathrm{i}, \mathrm{j} & \text { cell indices in } \mathrm{x} \text { and y direction } \\ L & \text { length scale } \\ p & \text { pressure } \\ T & \text { time scale } \\ u & \text { streamwise component of velocity } \\ v & \text { vertical component of velocity } \\ \operatorname{vol}(F) & \text { volume of fluid inside a cell }\end{array}$

\section{Greek symbol}

$\Delta t \quad$ time step

$\Delta x, \Delta y \quad$ grid size in $\mathrm{x}$ and $\mathrm{y}$ direction

\section{Non-dimensional numbers \\ $\mathrm{Fr}$ Froude number \\ Re Reynolds number \\ St Strouhal number}

\section{References}

[1] Liggett J A 1994 Fluid Mechanics. New York: McGraw-Hill
[2] Lighthill J 2001 Waves in fluids. South Asia edition: Cambridge University Press India

[3] Stoker J J 2011 Water waves: The mathematical theory with applications (Vol. 36). New York: John Wiley \& Sons

[4] Chanson H 2010 Undular tidal bores: basic theory and freesurface characteristics. Journal of Hydraulic Engineering 136: 940-944

[5] Martin J C, Moyce W J, Price A T and Thornhill C K 1952 Part IV. An experimental study of the collapse of liquid columns on a rigid horizontal plane. Philosophical Transactions of the Royal Society of London A, 244: 312-324

[6] Oertel M and Bung D B 2012 Initial stage of two-dimensional dam-break waves: laboratory versus VOF. Journal of Hydraulic Research 50: 89-97

[7] Chaudhry M H 2007 Open-channel flow. 2nd edition, New York: Springer Science \& Business Media

[8] Basco D 1987 Introduction to rapidly-varied unsteady, freesurface flow computation. US Geological Survey Water Resources Investigations Report 83-4284

[9] Mohapatra P K and Chaudhry M H 2004 Numerical solution of Boussinesq equations to simulate dam-break flows. Journal of Hydraulic Engineering 130: 156-159

[10] Pohle F V 1952 Motion of water due to breaking of a dam, and related problems. Proc. Gravity Waves 47

[11] Rao P 2002 Contribution of Boussinesq pressure and bottom roughness terms for open channel flows with shocks. Applied Mathematics and Computation 133: 581-590

[12] Castro-Orgaz O, Giráldez J V and Ayuso J L 2008 Higher order critical flow condition in curved streamline flow. Journal of Hydraulic Research 46: 849-853

[13] Hager W H 2010 Comments on "Steady open channel flows with curved streamlines: The Fawer approach revised". Environmental Fluid Mechanics 10: 491-494

[14] Devkota B H and Imberger J 2009 Lagrangian modeling of advection-diffusion transport in open channel flow. Water Resources Research 45

[15] Zobeyer H and Steffler P 2012 Modelling plane open-channel flows by coupled depth-averaged and RANS equations. Journal of Hydraulic Research 50: 82-88

[16] Harlow F H and Welch J E 1965 Numerical calculation of time-dependent viscous incompressible flow of fluid with free surface. Physics of Fluids 8: 2182-2189

[17] Amsden A A and Harlow F H 1970 A simplified MAC technique for incompressible fluid flow calculations. Journal of Computational Physics 6: 322-325

[18] Tome M F and McKee S 1994 GENSMAC: A computational marker and cell method for free surface flows in general domains. Journal of Computational Physics 110: 171-186

[19] Hirt C W and Nichols B D 1981 Volume of fluid (VOF) method for the dynamics of free boundaries. Journal of Computational Physics 39: 201-225

[20] Noh W F and Woodward P 1976 SLIC (simple line interface calculation). In: Proceedings of the fifth International Conference on Numerical Methods in Fluid Dynamics June 28-July 2, 1976 Twente University, Enschede (330-340). Springer.

[21] Youngs D L 1982 Time-dependent Multi-material Flow with Large Fluid Distortion. Numerical Methods for Fluid Dynamics 273-285

[22] Rudman M 1997 Volume-tracking methods for interfacial flow calculations. International Journal for Numerical Methods in Fluids 24: 671-691 
[23] Osher S and Sethian J A 1988 Fronts propagating with curvature-dependent speed: algorithms based on Hamilton-Jacobi formulations. Journal of Computational Physics 79: 12-49

[24] Sussman M, Smereka P and Osher S 1994 A level set approach for computing solutions to incompressible twophase flow. Journal of Computational Physics 114: 146-159

[25] Sussman M and Puckett E G 2000 A coupled level set and volume-of-fluid method for computing $3 \mathrm{D}$ and axisymmetric incompressible two-phase flows. Journal of Computational Physics 162: 301-337

[26] Olsson E and Kreiss G 2005 A conservative level set method for two phase flow. Journal of Computational Physics 210: 225-246

[27] Van der Pijl S P, Segal A, Vuik C and Wesseling P 2005 A mass-conserving Level-Set method for modelling of multiphase flows. International Journal for Numerical Methods in Fluids 47: 339-361

[28] Saha A K, Muralidhar K and Biswas G 2000 Transition and chaos in two-dimensional flow past a square cylinder. Journal of Engineering Mechanics 126: 523-532
[29] Hirt C W, Nichols B D and Romero N C 1975 SOLA: A numerical solution algorithm for transient fluid flows (No. LA-5852). Los Alamos Scientific Lab., N. Mex. (USA).

[30] Chorin A J 1967 A numerical method for solving incompressible viscous flow problems. Journal of Computational Physics 2: 12-26

[31] Mohapatra P K 1998 Studies of Two Dimensional Free Surface flows With Non-hydrostatic Pressure Distribution. Phd thesis, Dept of Civil Engineering IIT Kanpur

[32] Sohankar A, Norberg C and Davidson L 1998 Low-Reynolds-number flow around a square cylinder at incidence: study of blockage, onset of vortex shedding and outlet boundary condition. International Journal for Numerical Methods in Fluids 26: 39-56

[33] Chen S, Johnson D B and Raad P E 1995 Velocity boundary conditions for the simulation of free surface fluid flow. Journal of Computational Physics 116: 262-276

[34] Hirt C W and Shannon J P 1968. Free-surface stress conditions for incompressible-flow calculations. Journal of Computational Physics 2: 403-411 